\title{
A life course approach to total tooth loss: Testing the sensitive period, accumulation, and social mobility models in the Health and Retirement Study
}

\author{
Haena Lee
}

Survey Research Center, Institute for Social Research, University of Michigan, Ann Arbor, Michigan

\section{Correspondence}

Haena Lee, University of Michigan Institute for Social Research, 426 Thompson St, Ann Arbor, Ml 48104

Email: haenal@umich.edu

\section{Funding information}

This work is funded by the National Institute on Aging (grant number: R01AG051142) and the George E. Richmond Foundation through the University of Chicago Program in Oral Health, Systemic Health, Well-Being and the Social Sciences. The HRS (Health and Retirement Study) is sponsored by the National Institute on Aging (grant number: U01AG009740) and is conducted by the University of Michigan.

\begin{abstract}
Objectives: Childhood socio-economic status (SES) has long been associated with later-life oral health, suggesting that childhood is a sensitive period for oral health. Far less attention has been given to the long-term impact of childhood trauma, abuse, and smoking on later-life oral health. This study fills the gap in the literature by examining how adverse childhood experiences-social, psychological, and behavioralshape total tooth loss over the life course, with an assessment of the sensitive period, accumulation, and social mobility models from life course research.
\end{abstract}

Methods: Data are drawn from the 2012 Health and Retirement Study (HRS) merged with multiple HRS data sources to obtain childhood information ( $N=6,427$; age > 50). Adverse childhood experiences include childhood financial hardship, trauma, abuse, and smoking. Total tooth loss is measured to assess poor oral health in later life. Educational attainment and poverty status (since age 51 ) are measured as adult adversity. Current health conditions and health behaviors are assessed to reflect the correlates of oral health in later life.

Results: The sensitive period model indicates that childhood trauma such as parental death or divorce (odds ratio $[\mathrm{OR}]=1.37,95 \%$ confidence interval $[\mathrm{Cl}]=1.04,1.80$ ), physical abuse $(\mathrm{OR}=1.17,95 \% \mathrm{Cl}=1.03,1.34)$, and low educational attainment ( $\leq$ high school; $\mathrm{OR}=1.52,95 \% \mathrm{Cl}=1.04,2.22$ ) are associated with higher odds of total tooth loss in later life. Poverty status was not associated with the outcome. There was a clear graded relationship between accumulation of adverse experiences and oral health, which supports the accumulation model. In the social mobility model, older adults who occupied a stable disadvantageous position were more likely to be toothless $(\mathrm{OR}=1.77,95 \% \mathrm{Cl}=1.08,2.90)$ compared to those who did not face adversity in any case. Neither upward nor downward mobility mattered.

Conclusions: Failing oral health in older adults, especially total tooth loss, may have its roots in adverse experiences such as childhood trauma, abuse, and low educational attainment. Findings also suggest that oral health in later life may be more influenced by accumulation of adversity rather than changes in social and economic position over the life course. 


\section{1 | INTRODUCTION}

Oral diseases are one of the most common and expensive health problems to treat. ${ }^{1}$ Among other oral diseases, total tooth loss (edentulism) is considered a major public health problem in the United States, particularly for older adults. A recent report indicates nearly $20 \%$ of the population aged over 50 are edentulous. ${ }^{2}$ Retaining extant healthy teeth becomes critical in later life as it significantly affects "healthy aging," from functioning, ${ }^{3}$ to well-being, ${ }^{4}$ to longevity. ${ }^{5}$

Most of the previous work on this subject has identified adult socio-economic status (SES) ${ }^{6,7}$ and health conditions ${ }^{8}$ as key risk factors for tooth retention in adulthood. Only a few empirical studies have investigated the association between early-life adversities and oral health and focused primarily on socio-economic factors (e.g., financial hardship) as a key predictor that shapes oral health over time. ${ }^{9-11}$ Psychological and behavioral factors (e.g., childhood trauma, abuse, and smoking) have received little research attention, although their adverse associations with adult oral health have been well-documented. ${ }^{12}$ To date, there are only a few population-based studies of older adults containing current oral health status and retrospective childhood information. While the existing results are based on samples in the international contexts, ${ }^{9-11,13}$ how childhood experiences shape oral health among older adults in the United States remains largely overlooked. The current study contributes to this line of research by exploring the impact of childhood trauma, abuse, and smoking together with financial hardship in a nationally representative sample of older adults in the United States, and with attention to total tooth loss.

Conceptually, there are three models in life course research that explain the association between childhood experiences and health outcomes in later life. ${ }^{14}$ First, the sensitive period model suggests that later-life health has its origins during sensitive periods of development. In this model, childhood experiences are hypothesized to have a direct effect on health in later life, independent of adult experiences. ${ }^{14}$ The argument is that early stress induced by adverse childhood experiences may impair later-life health through "biological embedding," where early stress alters neurological response systems and damages immune systems. ${ }^{15}$ In the context of oral health, immune systems dysregulated by stressful experiences may increase susceptibilities to pathogenic bacteria and oral infection, potentially leading to tooth decay or periodontal diseases, ${ }^{16}$ one of the proximal causes of tooth loss. In a work by Boyce et al, ${ }^{17}$ children growing up in low-SES families are more likely to have dental caries in part due to higher levels of cariogenic oral bacteria which may damage the surface of enamel and thus increase the risk of dental caries. Early signs of dental caries are found to carry over into adult dental problems, ${ }^{18}$ providing a potential mechanism through which early stress may lead to poor tooth health over time. However, empirical evidence on the long-term relationships between stressful experiences in childhood and oral health at older ages is largely obscured.

Second, the accumulation model suggests that exposures to adversities at different life stages have a cumulative dose/response effect on health, increasing the risk of developing chronic disease. Adverse childhood experiences are considered to set in motion adult experiences that in turn influence health status. ${ }^{19}$ Childhood and adulthood adversities thus combine to yield lifelong profiles of social standing that can damage health. In this model, no one factor has a large impact on health, as poor adult health results from an accumulation of risk.

Third, an alternative approach for conceptualizing the accumulation of risk is the social mobility model. The social mobility model considers that the continuity and changes in adverse exposures between childhood and adulthood may affect adult health. In this model, those who remain in adverse conditions from childhood to adulthood are hypothesized to be in worse health than those who rise in their social position because it could compensate for early risk and lead to better health. ${ }^{11}$ Each of the three life course models may make unique contributions to understanding the potential pathways to adult health disparities, ${ }^{20}$ suggesting that testing the different models within the same setting may provide a more complete picture in studies of health, but this idea has not been thoroughly assessed for oral health. Only a few studies have empirically examined these models using international samples. ${ }^{21,22} \mathrm{~A}$ broader approach is much needed to better understand life course perspectives and their implication for oral health.

The aim of the current study is to determine the extent to which adverse childhood experiences impact total tooth loss among older adults in the United States. To determine whether the association between adverse experiences and total tooth loss is largely cumulative or specific to a certain period of time, the sensitive period, accumulation, and social mobility models are examined.

\section{2 | METHODS}

\section{1 | Data}

Data are drawn from the 2012 Health and Retirement Study (HRS), merged with multiple HRS data sources including the 2015 Life History Mail Survey (LHMS) and the retrospective childhood variables from the HRS core surveys (1998-2010). The HRS is a nationally representative longitudinal study of older adults in the United States and their spouses aged 51 and older, which includes the core surveyed every two years, and off-year supplementary mail surveys administered in between waves of the HRS core. ${ }^{23}$ The HRS was initiated in 1992 and has collected information on the changing social, financial, and health conditions of late adulthood in the core. Additionally, HRS has collected retrospective childhood information from 1998 onwards. Questions about childhood SES and health are included in the core entry interview, and from 2006 onwards, questions about early traumas are included in the psychosocial leave-behind questionnaire. The 2015 LHMS extended this information by asking detailed information on childhood family history. This study focuses on HRS respondents aged 51 and older in 2012 who were administered the supplemental survey in the 2015 LHMS ( $N=6,427)$. 
Outcome variable and most health-related variables came from the 2012 HRS core, while childhood experiences, adult educational attainment, and poverty status were drawn from the previous HRS surveys (1998-2010) and the 2015 LHMS.

\section{2 | Measures}

The outcome of interest in this study is total tooth loss. Total tooth loss was measured through respondent's report on the core question of, "Have you lost all your upper and lower natural permanent teeth?" (1 = yes; 0 = no).

Four binary indicators of childhood adversity were measured, including: (a) childhood financial hardship, (b) childhood abuse, (c) cigarette use during childhood, and (d) childhood trauma. Using the core, childhood financial hardship was created through the core question of, "Would you say your family, from birth to age 16, was pretty well off financially, about average, or poor?" ( 1 = poor; 0 = average/well off). Childhood abuse was measured if respondents reported in the leavebehind questionnaire that they were physically abused by parents ( $1=$ yes; $0=$ no). Childhood smoking was assessed through the question in the core, "Did you regularly (for six months or more) smoke cigarettes while you were in grade school or high school?" ( 1 = yes; 0 = no). Using the LHMS, childhood trauma was measured if respondents reported that, from birth to age 16 , they experienced parental death (either or both) and/or divorce (=1) compared with those who did not.

Two binary indicators of adult adversity were measured: (a) low educational attainment ( 1 = high school or less; 0 = college or more) and (b) poverty status since age 51. Poverty status was defined if a respondent's household income has ever been below the US Census poverty threshold since survey entry $(1=$ ever lived in poverty since age $51 ; 0=$ no). Because educational attainment is causally prior to poverty status, low educational attainment was considered adult adversity experienced in the period of early adulthood, while poverty status reflects late adulthood adversity.

Current heath behaviors and conditions were controlled to reflect correlates of poor oral health. Diabetes $(1=$ yes; $0=$ no) and lung diseases $(1=$ yes; $0=$ no) were adjusted as these are known contributors to poor oral health conditions such as periodontal diseases. ${ }^{24}$ Evidence suggests that memory performance is a strong predictor of oral hygiene. ${ }^{25}$ Memory performance was assessed by summing the number of immediate and delayed recall words. Health behaviors include recent dentist visit ( $1=$ yes in the past two years; $0=$ no), current smoking ( 1 = yes; $0=$ no), and drinking status (mild vs. heavy vs. no drinking [=reference]). Using the national guideline, ${ }^{26}$ heavy drinking was defined if respondents reported that they had more than three drinks when drinking (mild drinking = one or two drinks).

Sociodemographic covariates include age (range 51-99 in 2012), gender ( $1=$ female; 0 = male), race/ethnicity, and marital status ( 1 = married; 0 = not married). Respondents were grouped into four age brackets: (a) 51-60, (b) 61-71, (c) 71-80, and (d) 81+, to capture any differences across subgroups at older ages (i.e., young old, middle old, and oldest old). Race/ethnicity was self-reported and categorized into four groups: non-Hispanic White (=reference), non-Hispanic Black,
Hispanic, and others. In addition, self-reported childhood health was adjusted if respondents reported in the core that they had "fair" or "poor" health from birth to age 16 (=1; 0 = excellent/good), because such an answer may capture important early-life exposures, such as hospitalization and malnutrition, which were not assessed by the survey but may strongly correlate with poor oral health. ${ }^{27}$

\section{3 | Analytic strategy}

Because total tooth loss is a binary variable, logistic regression models were used in the current study. The first step was to estimate descriptive statistics and bivariate associations of key variables with total tooth loss. Next, multivariate logistic regressions were performed to examine the sensitive period and accumulation models. These models were accounted for current health conditions, current health behaviors, and sociodemographic covariates. Specifically, the sensitive period model was analysed by including adversity measures of childhood, early adulthood, and late adulthood in the same model. The accumulation model was investigated by combining the measures of the three periods into one measure. To do that, four indicators of childhood adversity were collapsed into a binary measure that represents any adverse experiences in childhood (vs. none). This binary variable was then combined with adversity measures in early and late adulthood, yielding a total of four combinations $(0,1,2$, and 3$)$. For the social mobility model, a four-category variable was created by summing the binary measures of adverse experiences from childhood to late adulthood: (a) stable advantageous; (b) upward mobility; (c) downward mobility; and (d) stable disadvantageous. This categorization was validated in prior work in life course research, ${ }^{21,22}$ and further details about the categorization are available in Table 3. Descriptive statistics and all analytical models were weighted using the survey data analysis commands (SVY) (Stata 15).

\section{3 | RESULTS}

Table 1 presents descriptive statistics for key variables and their bivariate associations with the outcome variable. Maintaining healthy teeth across the lifespan appears to be difficult for older adults, with over $13 \%$ of older adults over age 50 having lost all permanent teeth. Nearly $30 \%$ of respondents indicated that they had experienced financial hardship and traumatic events such as parental death or divorce by age $16(28.1 \%$ and $31.8 \%$, respectively). Physical abuse during childhood was not uncommon for this age cohort (10.6\%). A fair amount of respondents reported that they smoked during childhood (18\%). Almost $50 \%$ of respondents held a high school diploma or less. About $20 \%$ of respondents had lived in poverty at least once since age 51 . Sizable proportions of respondents reported that they had been diagnosed with diabetes and lung diseases (22.4\% and $9.5 \%$ ). Almost $70 \%$ of respondents visited a dentist in the past two years. More than $20 \%$ of respondents were current smokers (23\%), and about half of the respondents were mild or heavy drinkers $(52.3 \%$ and $3.7 \%$, respectively). 
TAB LE 1 Descriptive statistics and unadjusted odds ratios of total tooth loss by childhood, early adulthood, and late adulthood characteristics, Health and Retirement Study $2012(N=6,427)$

\begin{tabular}{|c|c|c|c|}
\hline & $\begin{array}{l}\% / \text { Mean } \\
\text { (SD) }\end{array}$ & $\begin{array}{l}\text { Unadjusted } \\
\text { OR }\end{array}$ & $95 \% \mathrm{Cl}$ \\
\hline Total tooth loss & $13.1 \%$ & - & - \\
\hline \multicolumn{4}{|c|}{ Life course adversity measures } \\
\hline \multicolumn{4}{|l|}{ Childhood } \\
\hline Financial hardship & $28.1 \%$ & $1.81^{* * *}$ & $1.37,2.38$ \\
\hline Trauma & $31.8 \%$ & $1.93^{* * *}$ & $1.52,2.46$ \\
\hline Abuse & $10.3 \%$ & $1.33^{*}$ & $1.03,2.06$ \\
\hline Cigarette use & $18.0 \%$ & $1.76^{* *}$ & $1.29,2.39$ \\
\hline \multicolumn{4}{|l|}{ Early Adulthood } \\
\hline $\begin{array}{l}\text { Low educational } \\
\text { attainment }\end{array}$ & $47.9 \%$ & $3.82^{* * *}$ & $2.81,5.19$ \\
\hline \multicolumn{4}{|l|}{ Late adulthood } \\
\hline $\begin{array}{l}\text { Ever lived in } \\
\text { poverty ( } \geq \text { age } 51 \text { ) }\end{array}$ & $20.5 \%$ & $2.42^{* * *}$ & $1.79,3.27$ \\
\hline \multicolumn{4}{|l|}{ Current health conditions } \\
\hline Diabetes & $22.4 \%$ & $1.42^{*}$ & $(1.07,1.87)$ \\
\hline Lung diseases & $9.5 \%$ & $3.04^{* * *}$ & $(2.27,4.08)$ \\
\hline $\begin{array}{l}\text { Memory } \\
\text { performance }\end{array}$ & $10.1(3.2)$ & $0.86^{* * *}$ & $(0.82,0.90)$ \\
\hline \multicolumn{4}{|l|}{ Current health behaviors } \\
\hline Dentist visit & $67.7 \%$ & $0.10^{* * *}$ & $(0.07,0.13)$ \\
\hline Current smoking & $23.0 \%$ & $3.54^{* * *}$ & $(2.30,5.43)$ \\
\hline \multicolumn{4}{|l|}{ Drinking (none = ref.) } \\
\hline Mild drinking & $52.3 \%$ & $0.36^{* * *}$ & $(0.27,0.48)$ \\
\hline Heavy drinking & $3.7 \%$ & 0.62 & $(0.36,1.09)$ \\
\hline \multicolumn{4}{|c|}{ Sociodemographic covariates } \\
\hline \multicolumn{4}{|l|}{ Age $(51-60=$ ref. $)$} \\
\hline $61-70$ & $29.5 \%$ & $1.79^{*}$ & $(1.14,2.80)$ \\
\hline $71-80$ & $27.7 \%$ & $2.59^{* * *}$ & $(1.81,3.70)$ \\
\hline+81 & $9.7 \%$ & $2.84^{* * *}$ & $(1.82,4.43)$ \\
\hline Female & $57.7 \%$ & 1.13 & $(0.88,1.45)$ \\
\hline \multicolumn{4}{|c|}{ Race/ethnicity (NH White = ref.) } \\
\hline NH Black & $7.6 \%$ & 1.47 & $(0.99,2.17)$ \\
\hline Hispanic & $5.2 \%$ & 1.48 & $(0.94,2.35)$ \\
\hline Other & $3.0 \%$ & 1.28 & $(0.53,3.08)$ \\
\hline Married & $63.7 \%$ & $0.51^{* * *}$ & $(0.40,0.66)$ \\
\hline $\begin{array}{l}\text { Childhood poor } \\
\text { health }\end{array}$ & $5.5 \%$ & 1.40 & $(0.81,2.42)$ \\
\hline
\end{tabular}

Abbreviation: $\mathrm{Cl}$, confidence interval; $\mathrm{NH}$, non-Hispanic; OR, odds ratio; ref., reference; $S D$, standard deviation.

${ }^{*} P<0.05$.

${ }^{* *} P<0.01$.

***P $<0.001$.

Bivariate associations indicate that adverse experiences throughout the lifespan and poor health conditions were each associated with increased odds of total tooth loss. Better memory performance, recent dentist visit, and mild drinking were protective of total tooth loss. This is not an unreasonable result as mild drinking is considered a healthy lifestyle. Indeed, mild drinkers may be more socially active and potentially sensitive to physical appearance, beneficial characteristics for retaining extant teeth. Odds of total tooth loss increased with age as expected, while marriage protected against total tooth loss (race/ethnicity and childhood poor health; n.s.).

Table 2 shows that the multivariate associations between life course adversities and total tooth loss risk under the sensitive period and accumulation models. In the sensitive period model, adverse experiences in childhood and early adulthood were both associated with increased odds of total tooth loss. Specifically, experiences of childhood trauma (odds ratio $[\mathrm{OR}]=1.37,95 \%$ confidence interval $[\mathrm{Cl}]=1.04$, 1.80), physical abuse (OR $=1.17,95 \% \mathrm{Cl}=1.03,1.34)$, and low educational attainment $(\mathrm{OR}=1.52,95 \% \mathrm{Cl}=1.04,2.22)$ were adversely associated with total tooth loss with all else held constant. Cigarette use in childhood was marginally associated with total tooth loss net of known risk factors including current smoking $(\mathrm{OR}=1.44,95 \% \mathrm{Cl}=0.96,2.17)$. In contrast, the association between poverty experience in later adulthood and total tooth loss was not statistically significant with the measured outcome $(O R=1.19,95 \% \mathrm{Cl}=0.84,1.69)$. For the accumulation model, the odds of total tooth loss increased with additional periods respondents experienced adversity. Compared with those who had never experienced adversity, experiencing one, two, and three periods of adversity yielded ORs of 2.65 ( $95 \% \mathrm{Cl}=1.55,4.55), 2.91$ (95\% $\mathrm{Cl}=1.57,5.41)$, and $4.06(95 \% \mathrm{Cl}=2.03,8.13)$, respectively.

To attempt to further explore the accumulation of risk and its impact on oral health, the social mobility model was examined as shown in Table 3. Findings from the social mobility model corroborated that those who persistently experienced adversities throughout life are most disadvantaged, with higher odds of total tooth loss for stable disadvantageous trajectory $(\mathrm{OR}=1.77,95 \% \mathrm{Cl}=1.08,2.90)$ compared to stable advantageous trajectory. Upward and downward mobility did not contribute to total tooth loss. Across all models, similar to results from bivariate associations, lung diseases, current smoking, and age increased the odds of total tooth loss, while memory performance and mild drinking were associated with lower odds of total tooth loss.

\section{4 | DISCUSSION}

To date, this is the first study to investigate the sensitive period, accumulation, and social mobility models in life course research, examining life course determinants of oral health of older adults in the United States. There was support for all three models, yielding three main points in this study. First, childhood and early adulthood are found to be sensitive periods during which individuals are particularly sensitive to risk factor exposures. Second, there was a graded linear relationship between the number of periods that individuals experienced adversity and oral health, corresponding to the accumulation model. Third, in the social mobility model, individuals persistently exposed to adversities from childhood to late adulthood are most disadvantaged compared to those who never faced adversities, pointing to the importance of continued risk exposure in oral health. 
TAB LE 2 Adjusted odds ratios and 95\% confidence intervals for risk of total tooth loss from the sensitive period model and accumulation model, Health and Retirement Study $2012(\mathrm{~N}=6,427)$

\begin{tabular}{|c|c|c|c|c|}
\hline & \multicolumn{2}{|c|}{ Sensitive period model } & \multicolumn{2}{|c|}{ Accumulation model } \\
\hline & OR & $95 \% \mathrm{Cl}$ & OR & $95 \% \mathrm{Cl}$ \\
\hline \multicolumn{5}{|l|}{ Life course adversity measures } \\
\hline \multicolumn{5}{|l|}{ Childhood } \\
\hline Financial hardship & 1.24 & $0.91,1.69$ & - & - \\
\hline Trauma & $1.37^{*}$ & $1.04,1.80$ & - & - \\
\hline Abuse & $1.17^{*}$ & $1.03,1.34$ & - & - \\
\hline Cigarette use & $1.44^{\dagger}$ & $0.96,2.17$ & - & - \\
\hline \multicolumn{5}{|l|}{ Adulthood } \\
\hline Low educational attainment & $1.52^{*}$ & $1.04,2.22$ & - & - \\
\hline \multicolumn{5}{|l|}{ Late adulthood } \\
\hline Ever lived in poverty ( $\geq$ age 51 ) & 1.19 & $0.84,1.69$ & - & - \\
\hline \multicolumn{5}{|c|}{ Number of periods experiencing social adversities over the life course } \\
\hline 0 period & - & - & 1.00 & - \\
\hline 1 periods & - & - & $2.65^{* * *}$ & $1.55,4.55$ \\
\hline 2 periods & - & - & $2.91^{* *}$ & $1.57,5.41$ \\
\hline 3 periods & - & - & $4.06^{* * *}$ & $2.03,8.13$ \\
\hline \multicolumn{5}{|l|}{ Current health conditions } \\
\hline Diabetes & 1.08 & $0.76,1.53$ & 1.05 & $0.76,1.45$ \\
\hline Lung diseases & $1.80^{* *}$ & $1.24,2.61$ & $1.77^{* *}$ & $1.27,2.46$ \\
\hline Memory performance & $0.95^{\dagger}$ & $0.90,1.01$ & $0.95^{\dagger}$ & $0.90,1.00$ \\
\hline \multicolumn{5}{|l|}{ Current health behaviors } \\
\hline Dentist visit & $0.13^{* * *}$ & $0.09,0.19$ & $0.14^{* * *}$ & $0.10,0.20$ \\
\hline Current smoking & $2.36^{* *}$ & $1.38,4.02$ & $2.50^{* * *}$ & $1.58,3.95$ \\
\hline \multicolumn{5}{|l|}{ Drinking (none = ref.) } \\
\hline Mild drinking & $0.60^{* *}$ & $0.44,0.82$ & $0.64^{* *}$ & $0.47,0.86$ \\
\hline Heavy drinking & 0.64 & $0.31,1.31$ & 0.74 & $0.37,1.45$ \\
\hline \multicolumn{5}{|l|}{ Sociodemographic covariates } \\
\hline \multicolumn{5}{|l|}{ Age $(51-60=$ ref.) } \\
\hline $61-70$ & $1.65^{*}$ & $1.06,2.57$ & $1.59^{*}$ & $1.10,2.29$ \\
\hline $71-80$ & $1.92^{* *}$ & $1.22,3.00$ & $1.91^{* * *}$ & $1.38,2.64$ \\
\hline+81 & $2.44^{* * *}$ & $1.42,4.22$ & $2.58^{* * *}$ & $1.66,4.01$ \\
\hline Female & 1.17 & $0.89,1.53$ & 1.14 & $0.89,1.47$ \\
\hline \multicolumn{5}{|l|}{ Race/ethnicity (NH White = ref.) } \\
\hline NH Black & 1.17 & $0.78,1.75$ & 1.17 & $0.78,1.74$ \\
\hline Hispanic & 1.31 & $0.74,2.32$ & 1.19 & $0.71,2.00$ \\
\hline Other & 1.82 & $0.74,4.50$ & 1.43 & $0.59,3.48$ \\
\hline Married & 0.76 & $0.54,1.08$ & 0.85 & $0.63,1.14$ \\
\hline Childhood poor health & 0.84 & $0.45,1.57$ & 0.87 & $0.51,1.49$ \\
\hline F-statistics & $19.70^{* * *}$ & & $16.25^{* * *}$ & \\
\hline
\end{tabular}

Abbreviation: $\mathrm{Cl}$, confidence interval; $\mathrm{NH}$, non-Hispanic; OR, odds ratio; Ref., reference group. ${ }^{\dagger} P<0.10$.

${ }^{*} P<0.05$

${ }^{* *} P<0.01$.

${ }^{* * *} P<0.001$

The current study identified that childhood and early adulthood are sensitive periods in which oral health decrements take root. What might explain the associations between childhood trauma, childhood abuse, and low educational attainment and tooth loss in later life? First, childhood abuse may cumulatively affect total tooth loss through sociobehavioral pathways. Abused 
TAB LE 3 Adjusted odds ratios and 95\% confidence intervals for risk of total tooth loss from the social mobility model, Health and Retirement Study 2012 ( $N=6,427)$

\begin{tabular}{|lll} 
& \multicolumn{2}{l}{ Social mobility model } \\
\cline { 2 - 3 } & OR & $95 \% \mathrm{Cl}$ \\
\hline Lifetime adversity patterns & & \\
\hline Stable advantageous & 1.00 & - \\
\hline Upward mobility & 1.27 & $0.93,1.72$ \\
\hline Downward mobility & 1.13 & $0.65,1.98$ \\
\hline Stable disadvantageous & $1.77^{*}$ & $1.08,2.90$ \\
\hline F-statistics & $22.79^{* *}$ & \\
\hline
\end{tabular}

Note: Stable advantageous denotes no adverse experiences over the life course. Upward mobility denotes adversity experienced in childhood but not in adulthood. Downward mobility denotes no adversity in childhood but experienced in adulthood. Stable disadvantageous denotes persistently experienced adversity over the life course. Table 3 controls for current health conditions, current health behaviors, and sociodemographic covariates.

Abbreviation: $\mathrm{Cl}$, confidence interval; OR, odds ratio.

${ }^{*} P<0.05$

${ }^{* * *} P<0.001$

adolescents, for example, may be at high risk of traumatic dental injuries in adulthood if they subsequently develop behavioral problems such as being aggressive and violent as a result of early exposures to family violence. ${ }^{28}$ They may also be more likely to engage in maladaptive health behaviors in adulthood such as binge drinking in response to early stressful circumstances. Excessive consumption of sugar from alcohol and nicotine is a major cause of tooth decay, for which the result contributes to tooth loss risk. Second, childhood trauma may be associated with tooth loss through stress mechanisms. Evidence suggests that early stress may damage oral health through its impact on inhibitory control system of the brain which, if damaged, may perpetuate addictive behaviors such as nicotine dependence, ${ }^{29}$ the result of which may lead to tooth loss. Third, low educational attainment in adulthood may also affect oral health through socio-economic pathways. Individuals with low educational attainment may be less likely to have occupations that come with good health benefits, which may result in underutilization of preventive care services. ${ }^{30}$ Educational attainment appears to have an impact on health literacy, and individuals with low health literacy appear to be more likely to delay getting care. ${ }^{31}$ Similar results are revealed in a supplementary analysis, with a lower likelihood of visiting a dentist among poorly educated respondents (results not shown).

One of the strengths of this study is that it extends the concept of adverse childhood experiences by incorporating not only childhood financial hardship, but also various types of early adversities such as parental death, divorce, abuse, and early cigarette use. Family trauma and dysfunction have long been considered, independent of childhood SES, to be strongly and negatively associated with a host of later-life health outcomes; however, little has been known of their effects on oral health in later life. The findings-the significant effect of parental death, divorce, and physical abuse on total tooth lossclarify that childhood trauma and abuse are yet another instance of important childhood factors that cumulatively damage health over the life course. A recent study ${ }^{32}$ conducted among Japanese older adults found that childhood abuse affects tooth retention, supporting our findings and suggesting that exploring stressful experiences during childhood merits attention to understand the full impact of these experiences on oral health. The current study did not find the significant association between childhood financial hardship and total tooth loss. It may be the case that individuals may be able to fix teeth (ie, through implants and tooth reconstruction surgery) if they overcome childhood financial difficulties with the amassing of resources in adulthood. Future studies investigating the potential pathways through which childhood SES shapes dental care services would be beneficial. Another strength is that the current study expanded the previous work by investigating three life course models in the context of oral health. Findings support all three models, indicating their complementary relationships. Some studies argue that these models are often mutually inclusive,$^{14}$ making it difficult to distinguish which models best describe the link between early-life adversities and health outcomes. ${ }^{20,33} \mathrm{~A}$ more nuanced approach, perhaps a simultaneous test of life course hypotheses, ${ }^{34}$ will help parse out the complex, underlying processes behind this oral health disparity.

There are several limitations in this study. First, the only measure of oral health that the HRS core collects is total tooth loss. The HRS core does not include measures of either the number of remaining teeth or which teeth respondents have lost. To some degree, being completely edentulous is a better situation than having some or many missing teeth, because the former can be treated easily with dentures. However, the HRS core does not include data on dentures. Second, because the measures of childhood experiences are retrospective, they may be subject to recall bias. The related results must be interpreted with caution. Third, detailed information on the onset of total tooth loss is not available in the HRS. Losing all teeth by midlife would affect an individual's life chances and outcomes differently than losing all teeth late in life. More detailed information on the pacing and sequencing of tooth loss would be beneficial. Lastly, other variables such as periodontal diseases or oral hygiene behaviors may be related to total tooth loss risk, but this information was not available in the HRS core. Despite the limitations, the current study is the first study to elucidate the relationship between adverse childhood experiences and oral health later in life using a representative sample of adults in the United States.

In conclusion, preventing childhood abuse and trauma should be considered in planning appropriate preventive measures and interventions to improve childhood well-being and parent-child relationships that may in turn help reduce oral health disparities in the long run. The current study also provides evidence that low educational attainment is a significant predictor of total tooth loss. Public health programs aimed at promoting health literacy would be beneficial in increasing access to preventive dental care services, especially for those with low levels of education. Policies and programs aiming at reducing cumulative exposures to adversities 
throughout life would narrow oral health gaps by fostering early intervention and facilitating appropriate preventive treatment.

\section{ORCID}

Haena Lee (iD https://orcid.org/0000-0001-5061-2564

\section{REFERENCES}

1. Petersen PE, Bourgeois D, Ogawa H, Estupinan-Day S, Ndiaye C. The global burden of oral diseases and risks to oral health. Bull World Health Organ. 2005;83(9):661-669.

2. Wu B, Liang J, Plassman BL, Remle RC, Luo X. Edentulism trends among middle-aged and older adults in the United States: comparison of five racial/ethnic groups. Community Dent Oral Epidemiol. 2012;40(2):145-153.

3. Kaye EK, Valencia A, Baba N, Spiro A, Dietrich T, Garcia RI. Tooth loss and periodontal disease predict poor cognitive function in older men. J Am Geriatr Soc. 2010;58(4):713-718.

4. Rouxel P, Tsakos G, Chandola T, Watt RG. Oral health-a neglected aspect of subjective well-being in later life. J Gerontol Ser B. 2018;73(3):382-386.

5. Ansai T, Takata Y, Soh I, et al. Relationship between tooth loss and mortality in 80-year-old Japanese community-dwelling subjects. BMC Public Health. 2010;10(1):1.

6. Gilbert GH, Duncan RP, Shelton BJ. Social determinants of tooth loss. Health Serv Res. 2003;38(6 Pt 2):1843-1862.

7. Matsuyama Y, Aida J, Tsuboya T, et al. Are lowered socioeconomic circumstances causally related to tooth loss? a natural experiment involving the 2011 great East Japan earthquake. Am J Epidemiol. 2017;186(1):54-62.

8. Al-Shammari KF, Al-Khabbaz AK, Al-Ansari JM, Neiva R, Wang $\mathrm{H}$-L. Risk indicators for tooth loss due to periodontal disease. $J$ Periodontol. 2005;76(11):1910-1918.

9. Listl S, Watt RG, Tsakos G. Early life conditions, adverse life events, and chewing ability at middle and later adulthood. Am J Public Health. 2014;104(5):e55-e61.

10. Fantin R, Delpierre C, Kelly-Irving M, Solís CB. Early socioeconomic conditions and severe tooth loss in middle-aged Costa Ricans. Community Dent Oral Epidemiol. 2018;46(2):178-184.

11. Poulton R, Caspi A, Milne BJ, et al. Association between children's experience of socioeconomic disadvantage and adult health: a lifecourse study. The Lancet. 2002;360(9346):1640-1645.

12. Puterman E, Gemmill A, Karasek D, et al. Lifespan adversity and later adulthood telomere length in the nationally representative US Health and Retirement Study. Proc Natl Acad Sci USA. 2016;113(42):E6335-E6342.

13. Nicolau B, Netuveli G, Kim J-WM, Sheiham A, Marcenes W. A lifecourse approach to assess psychosocial factors and periodontal disease. J Clin Periodontol. 2007;34(10):844-850.

14. Ben-Shlomo Y, Kuh D. A life course approach to chronic disease epidemiology: conceptual models, empirical challenges and interdisciplinary perspectives. Int J Epidemiol. 2002;31(2): 285-293.

15. Gluckman PD, Hanson MA, Cooper C, Thornburg KL. Effect of in utero and early-life conditions on adult health and disease. N Engl J Med. 2008;359(1):61-73.

16. Genco RJ, Ho AW, Kopman J, Grossi SG, Dunford RG, Tedesco LA. Models to evaluate the role of stress in periodontal disease. Ann Periodontol. 1998;3(1):288-302.

17. Boyce WT, Den Besten PK, Stamperdahl J, et al. Social inequalities in childhood dental caries: The convergent roles of stress, bacteria and disadvantage. Soc Sci Med. 2010;71(9):1644-1652.
18. Lu HX, Wong M, Lo E, McGrath C. Trends in oral health from childhood to early adulthood: a life course approach. Community Dent Oral Epidemiol. 2011;39(4):352-360.

19. Dannefer D. Cumulative advantage/disadvantage and the life course: cross-fertilizing age and social science theory. J Gerontol Ser B. 2003;58(6):S327-S337.

20. Hallqvist J, Lynch J, Bartley M, Lang T, Blane D. Can we disentangle life course processes of accumulation, critical period and social mobility? An analysis of disadvantaged socio-economic positions and myocardial infarction in the Stockholm Heart Epidemiology Program. Soc Sci Med. 2004;58(8):1555-1562.

21. ThekkePurakkal AS, Naimi AI, Madathil SA, et al. Differential impact of socioeconomic position across life on oral cancer risk in Kerala, India: an investigation of life-course models under a time-varying framework. Community Dent Oral Epidemiol. 2018;46(6):592-600.

22. Bernabé E, Suominen AL, Nordblad A, et al. Education level and oral health in Finnish adults: evidence from different lifecourse models: lifecourse models and oral health. J Clin Periodontol. 2011;38(1):25-32.

23. Sonnega A, Faul JD, Ofstedal MB, Langa KM, Phillips JW, Weir DR. Cohort profile: the health and retirement study (HRS). Int J Epidemiol. 2014;43(2):576-585.

24. Pihlstrom BL, Michalowicz BS, Johnson NW. Periodontal diseases. The lancet. 2005;366(9499):1809-1820.

25. Wu B, Fillenbaum GG, Plassman BL, Guo L. Association between oral health and cognitive status: a systematic review. J Am Geriatr Soc. 2016;64(4):739-751.

26. National Institute on Alcohol Abuse and Alcoholism. Alcohol \& Your Health: Older Adults. Available from URL: https://www.niaaa.nih.gov/ alcohol-health/special-populations-co-occurring-disorders/older-adults.

27. Patrick DL, Lee R, Nucci M, Grembowski D, Jolles CZ, Milgrom P. Reducing oral health disparities: a focus on social and cultural determinants. BMC Oral Health. 2006;6(1):1.

28. Nicolau B, Thomson WM, Steele JG, Allison PJ. Life-course epidemiology: concepts and theoretical models and its relevance to chronic oral conditions. Community Dent Oral Epidemiol. 2007;35(4):241-249.

29. Elliott JC, Stohl M, Wall MM, et al. The risk for persistent adult alcohol and nicotine dependence: the role of childhood maltreatment. Addict Abingdon Engl. 2014;109(5):842-850.

30. Sabbah W, Tsakos G, Sheiham A, Watt RG. The role of health-related behaviors in the socioeconomic disparities in oral health. Soc Sci Med. 2009;68(2):298-303.

31. Levy $\mathrm{H}$, Janke A. Health literacy and access to care. J Health Commun. 2016;21(Suppl):43-50.

32. Matsuyama Y, Fujiwara T, Aida J, et al. Experience of childhood abuse and later number of remaining teeth in older Japanese: a lifecourse study from Japan Gerontological Evaluation Study project. Community Dent Oral Epidemiol. 2016;44(6):531-539.

33. Rosvall M, Chaix B, Lynch J, Lindström M, Merlo J. Similar support for three different life course socioeconomic models on predicting premature cardiovascular mortality and all-cause mortality. BMC Public Health. 2006;6(1):203.

34. Darin-Mattsson A, Andel R, Celeste RK, Kåreholt I. Linking financial hardship throughout the life-course with psychological distress in old age: Sensitive period, accumulation of risks, and chain of risks hypotheses. Soc Sci Med. 2018;201:111-119.

How to cite this article: Lee $\mathrm{H}$. A life course approach to total tooth loss: Testing the sensitive period, accumulation, and social mobility models in the Health and Retirement Study. Community Dent Oral Epidemiol. 2019;47:333-339. https://doi. org/10.1111/cdoe.12463 